\title{
Coupling of high temperature nuclear reactor with chemical plant by means of steam loop with heat pump
}

\author{
Mariusz Kopeć ${ }^{1, *}$ \\ ${ }^{1}$ AGH-University of Science and Technology, Faculty of Energy and Fuels, Kraków, Poland
}

\begin{abstract}
High temperature nuclear reactors (HTR) can be used as an excellent, emission-free source of technological heat for various industrial applications. Their outlet helium temperature $\left(700^{\circ}-900^{\circ} \mathrm{C}\right)$ allows not only for heat supply to all processes below $600^{\circ} \mathrm{C}$ (referred to as "steam class"), but also enables development of clean nuclear-assisted hydrogen production or coal liquefaction technologies with required temperatures up to $900^{\circ} \mathrm{C}$ (referred to as "chemical class"). This paper presents the results of analyses done for various configurations of the steam transport loop coupled with the high-temperature heat pump designed for "chemical class" applications. The advantages and disadvantages as well as the key issues are discussed in comparison with alternative solutions, trying to answer the question whether the system with the steam loop and the hightemperature heat pump is viable and economically justified.
\end{abstract}

\section{HTR as a source of process heat}

According to the nomenclature proposed in the EUROPAIRS Project [1], industrial heat consumers can be grouped into the following 3 classes:

- steam class $\left(\sim 150^{\circ} \mathrm{C}\right.$ up to $\left.\sim 600^{\circ} \mathrm{C}\right)$ where steam is used both to the heat transport and heating processes. E.g. desalination, drying, paper industry, chemical industry (soda ash, ammonia and urea), production of plastic, non-ferrous metals, refineries.

- chemical class $\left(\sim 600^{\circ} \mathrm{C}\right.$ up to $\left.\sim 900^{\circ} \mathrm{C}\right)$ where heat is mainly consumed as the driver of chemical reactions delivering required reaction enthalpy. E.g. production of plastic, hydrogen, coal liquefaction.

- mineral class (above $\sim 1000^{\circ} \mathrm{C}$ ) where heat is used to melt solids or to drive reactions between solids. E.g. production of lime, cement, ore sintering, coke making, metallurgy. The industrial processes belonging to these classes usually generate waste heat with temperatures enough to be used for washing, rinsing, food preparation or even district heating. In the conventional technologies the required heat is generated almost exclusively by the combustion of fossil fuels. However, the burning of fossil fuels on the global scale raises serious environmental concerns, as it is the largest source of greenhouse gases emissions, mainly $\mathrm{CO} 2$. These concerns are the most important motivation for the search for alternative sources of clean process heat.

\footnotetext{
*Corresponding author: Mariusz.Kopec@agh.edu.pl
} 
The high temperature nuclear reactor (HTR) can serve as an excellent, emission-free source of technological heat for various industrial applications, combining high efficiency with advanced safety features. The coolant temperature on HTR outlet can be as high as $900^{\circ} \mathrm{C}$ to $1000^{\circ} \mathrm{C}$ (for VHTR even $1200^{\circ} \mathrm{C}$ ), but the temperature available for the processes is limited rather by parameters of the heat transport loop. Within the EUROPAIRS Project the following three configurations have been considered:

- steam generator and the steam based heat transport loop;

- gas-gas Intermediate Heat eXchanger and the gas based heat transport loop;

- gas-molten salt IHX and the molten salt based heat transport loop.

The use of steam transport loop is the most mature and proven technology. With existing technologies this coupling can deliver process steam at temperatures up to $600^{\circ} \mathrm{C}$ (or even more). All limitations are related to the resistance to creep, fatigue and corrosion of the applicable high-temperature materials, and appear virtually the same as in conventional supercritical steam systems. The near-term coupling scheme proposed in EUROPAIRS Project (with one HTR core) is shown in Fig. 1.

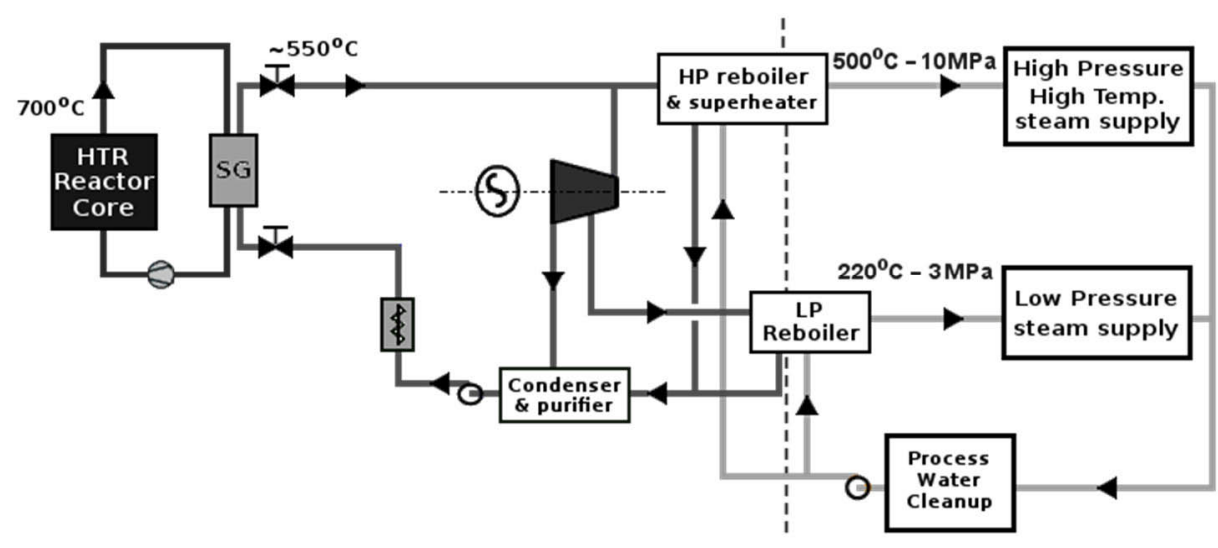

Fig. 1. System configuration proposed in EUROPAIRS Project

The gas-gas transport loop could provide heat at temperatures around $750^{\circ} \mathrm{C}$ to $800^{\circ} \mathrm{C}$, which may be sufficient for some chemical class end-users. However, this technology cannot be regarded as ready to use because at these temperatures there are still challenges concerning applicable materials capable of withstanding creep damage for long lifetimes. Further development of appropriate heat exchangers is also required. Additionally, it must be taken into account that in a real situation some "safety distance" between the reactor and the chemical plant will be required. In such a case the hot helium would have to be transported over a long distance. Beside the high cost of helium, the material issues at these temperatures would require use of very expensive materials, increasing dramatically the expected installation cost.

Comparing to the gas loop, the loop on molten salts would allow for lower pressures, higher power densities and more efficient heat transfer. However, due to serious corrosion problems, high pressure drop and high risk of freezing, this configuration would require intensive $\mathrm{R} \& \mathrm{D}$ and probably cannot be consider even as the medium term option.

The positive long-term experience with HTRs and analysis of possible coupling schemes indicate that the current technological maturity is sufficient to deploy these reactors as an emission-free source of process heat for steam class end-users. Simultaneously it is clear that the HTR cannot be used as the heat source for the mineral class applications because the temperatures on reactor outlet are too low. But in case of 
chemical class applications, the situation is more complex. Direct heating by means of gasgas IHX and the gas heat transport loop is indeed possible as a medium-term option, but the near term solution can utilize the proven and relatively inexpensive steam loop technology with one additional step in which the temperature is raised to the level required by the endusers. At the simplest version it can be carried out by external heating (e.g. electric), but some authors suggest that higher efficiency can be achieved with a mechanical hightemperature heat pump [2].

\section{Nuclear heat supply system for chemical class end-users}

The steam-based nuclear heat supply system for chemical class end-users generally follows the configuration proposed in EUROPAIRS Project for the steam class coupling. The helium on HTR outlet has a temperature of $700^{\circ} \mathrm{C}$ and the steam leaves steam generator $(\mathrm{SG})$ at $550^{\circ} \mathrm{C}$ and $10 \mathrm{MPa}$. The steam is transported over the long loop configured as a classical bottoming-cycle $\mathrm{CHP}$, where the heat at $\sim 530^{\circ} \mathrm{C}$ is directed through the heat exchanger towards the end-user installation, and the waste heat is used for power generation. As the temperature $530^{\circ} \mathrm{C}$ is too low for chemical class applications, in the next step it must be raised to the required level which in the presented analyzes was set to $850^{\circ} \mathrm{C}$. The steam leaving the $\mathrm{HX}$ is expanded in a steam turbine providing mechanical work which can be used directly or converted into electrical energy, subsequently required to raise the output temperature. The latter configuration offers higher flexibility and has been assumed as the reference for the purposes of further analyzes. For simplicity, any losses due to conversion between mechanical work and electrical energy are further neglected.

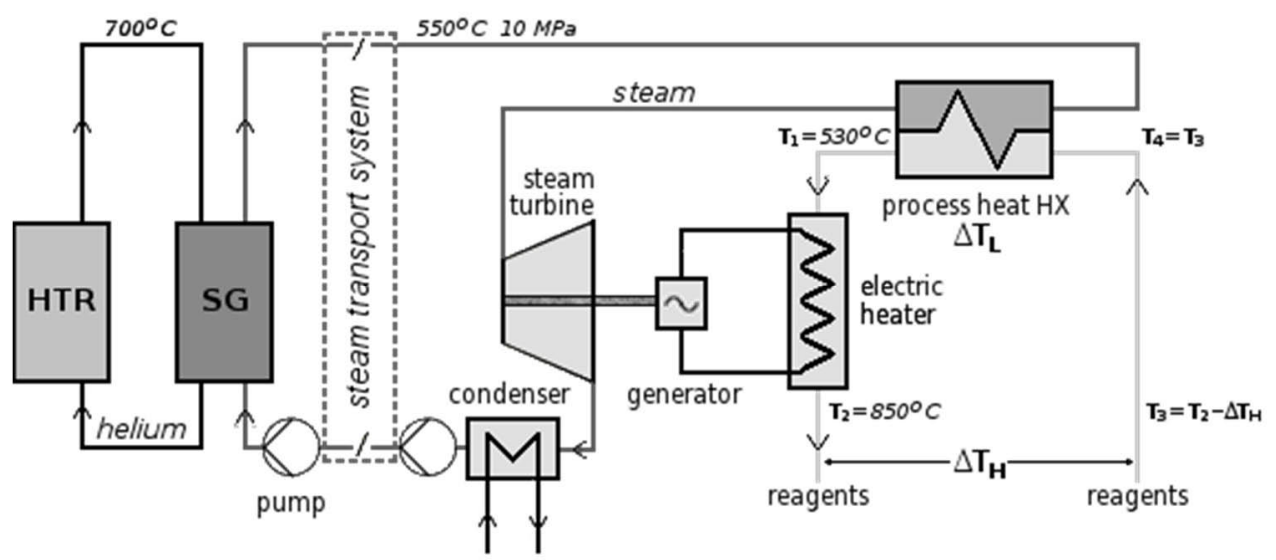

Fig. 2. Configuration of the heat transport system with an electrical heater

The additional step is necessary to increase the output temperature from $530^{\circ} \mathrm{C}$ to $850^{\circ} \mathrm{C}$. This can be accomplished by simple electrical heating or by using the mechanical heat pump. The first configuration, shown in Fig. 2, is very simple. It is based on existing technologies and can be deployed almost immediately, but the total efficiency will be limited by efficiency of electricity production. The second one, shown in Fig. 3, will be much more complicated (and expensive), but the total efficiency can be higher than in the first case. For simplicity the same $\Delta \mathrm{T}_{\mathrm{H}}$ has been assumed for both streams in HTHX, and simultaneously the temperature drop between gas and heated medium has been set at $20^{\circ} \mathrm{C}$. 


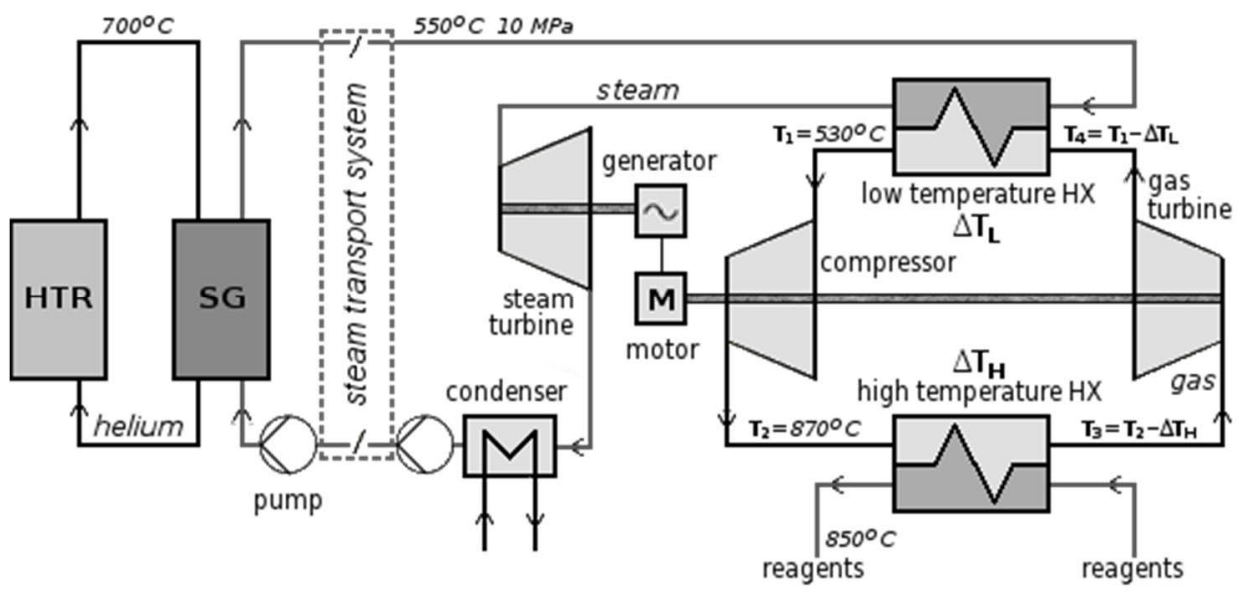

Fig. 3. Configuration of the heat transport system with a mechanical heat pump

It should be noted that regardless of the considered system configuration, most of its parameters will be determined by four basic factors:

- $\mathrm{T}_{\mathrm{L}}$ - the temperature retrieved from the $\mathrm{HX}$ on steam loop - here $\mathrm{T}_{\mathrm{L}}=\mathrm{T}_{1}=530^{\circ} \mathrm{C}$

- $\mathrm{T}_{\mathrm{H}}$ - the gas temperature after compression - here $\mathrm{T}_{\mathrm{H}}=\mathrm{T}_{2}=870^{\circ} \mathrm{C}$

- $\Delta \mathrm{T}_{\mathrm{H}}$ - the temperature span at which the end-user consumes the heat supplied.

- $\eta_{\mathrm{e}}$ - efficiency of electricity production.

In the chemical class the high-temperature heat is mainly used as a driver of endothermic chemical reactions, and is usually consumed at almost constant temperature. It means that this temperature span will be rather narrow, and it is safe to assume that in most situations $\Delta \mathrm{T}_{\mathrm{H}}<200^{\circ} \mathrm{C}$.

Operating experience with the high temperature nuclear reactors shows that the efficiency of electricity production in the Rankine cycle is lower than $40 \%$ (e.g. Peach Bottom $1-34.8 \%$, Fort St.Vrain - 39.2\%, THTR - 39.5\%) [3]. The steam parameters used in this work $\left(550^{\circ} \mathrm{C} @ 10 \mathrm{MPa}\right)$ are the most close to those in Peach Bottom reactor $\left(538^{\circ} \mathrm{C}\right.$ (a) $10 \mathrm{MPa}$, no reheat) [4] whereas the other reactors used higher steam pressures (16.6 and 18.6 $\mathrm{MPa}$ respectively) as well as steam reheat. For this reason, it is justified to use in analyzes $\eta_{\mathrm{e}}=35 \%$ what is very close to the efficiency of Peach Bottom reactor. In addition, it should be noted that in case of heat transport system the steam turbine will work in the bottoming cycle so the efficiency of electricity production will be lower than it would be for the fresh steam.

\subsection{Key indicators of system performance}

In presented analyses it is assumed that the whole energy passed to the end-user installation in form of high-temperature heat is produced by the same high temperature reactor. The high temperature heat supply systems will use the low temperature heat flux $\dot{Q}_{L}$ retrieved from LT HX (or process HX) and the mechanical power $\dot{W}_{M}$ to pass the high temperature heat flux $\dot{Q}_{H}$. It should be noted that the temperatures called here "low" are in fact around $530^{\circ} \mathrm{C}$ whereas the "high temperatures" may be well above $850^{\circ} \mathrm{C}$. The energy balance is as follows:

$$
Q_{H}=Q_{L}+W_{M}
$$


Both electricity and heat come from the same source, but the former is generated with a low efficiency $\eta_{\mathrm{e}}=0.35$. Therefore the overall efficiency is defined as:

$$
\eta=\frac{Q_{H}}{Q_{L}+W_{M} / \eta_{e}}
$$

The next two indicators of interest are the share of the heat used:

$$
R_{Q L}=Q_{L} / Q_{H}
$$

and the share of mechanical work used:

$$
R_{W M}=W_{M} / Q_{H}
$$

This last value determines also the overall efficiency:

$$
\eta=\frac{1}{1+R_{W M}\left(1 / \eta_{e}-1\right)}
$$

From the point of view of system optimization the value of Rwm should be as low as possible, because the power, generated at a relatively low efficiency, is more expensive than heat. Low value of Rwm corresponds to a high value of RQL which simply means that the heat passed from the steam loop is used effectively. However, it is important that both heats must be positive. A negative value indicates that the heat is transported in the opposite direction.

\subsection{Electric heating}

The small temperature drop in the chemical loop $\left(\Delta \mathrm{T}_{\mathrm{H}}\right)$ has serious consequences for the system shown in Fig. 2, because:

$$
\Delta T_{L}=\Delta T_{H}+\left(T_{1}-T_{2}\right)=\Delta T_{H}-320^{\circ} \mathrm{C}
$$

and when $\mathrm{T}_{1}=530^{\circ} \mathrm{C}$ and $\mathrm{T}_{2}=850^{\circ} \mathrm{C}$ the process $\mathrm{HX}$ would work inversely, passing heat from the chemical loop to the steam loop always when $\Delta \mathrm{T}_{\mathrm{H}}<320^{\circ} \mathrm{C}$. This means that in practice this heat exchanger never will transfer heat to the chemical loop and therefore is not needed, as well as the whole heat transport loop. The entire high-temperature heat required by the end-user will be supplied exclusively by the electric heater, and the electric power may be generated on site of the HTR power plant. The system gets really simple and this configuration will be used as the reference one.

Substitution of $Q_{L}=0$ in equations (1)-(4) gives:

- $\eta=\eta_{\mathrm{e}}=0.35$

- $\mathrm{R}_{\mathrm{WM}}=1.0$

- $\mathrm{R}_{\mathrm{QL}}=0.0$

In fact, this configuration does not even require the use of high-temperature reactors, since electricity can be provided by any source. However, the zero-emission requirement imposes restrictions on the method for producing electricity. The deployment of electric heating does not require intensive $R \& D$ and this solution can be used almost at once. 


\subsection{High-temperature heat pump}

A heat pump is a device that uses external power to transfers heat from one fluid at low temperature to another one at higher temperature. Heat pumps are commonly used at much lower temperatures, transforming worthless waste heat to useful heat of higher temperature. Performance of conventional heat pumps is described by the ratio of heat supplied to the work consumed (COP), which often exceeds 1 . However, this parameter cannot be used for high-temperature heat pumps, because in their case there is no worthless heat and instead of COP the overall efficiency (2) will be used.

\subsubsection{Heat pump without regeneration}

The described here high-temperature heat pump consists of a gas compressor, a gas turbine and two heat exchangers (LT and HT). The compressor and the turbine are mounted on the same shaft, and are driven by an electric motor. In terms of thermodynamics, this device implements a reverse Brayton cycle, which for reversible processes is shown in Fig. 4.
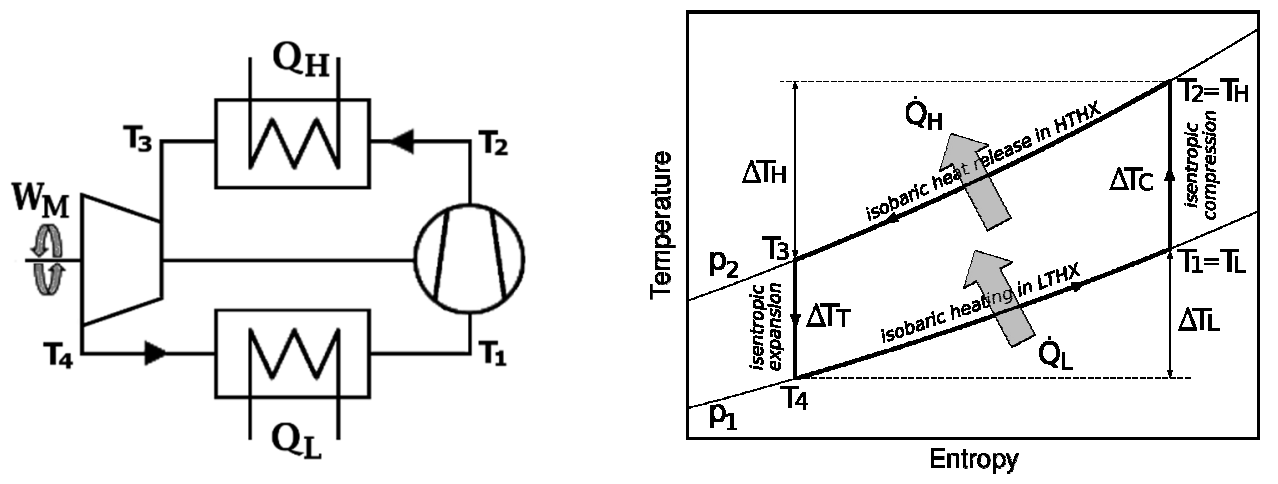

Fig. 4. Simple HT heat pump and the reverse Brayton cycle for reversible processes

It should be noted that the temperatures $\mathrm{T}_{\mathrm{L}}$ and $\mathrm{T}_{\mathrm{H}}$ are always set by the external conditions, and must be the same in all cycles analyzed.

\section{Reversible processes}

In case of an ideal gas there are two adiabatic processes:

$$
T_{2} p_{2}^{\left(\frac{1-\kappa}{\kappa}\right)}=T_{1} p_{1}^{\left(\frac{1-\kappa}{\kappa}\right)} \text { and } T_{3} p_{2}^{\left(\frac{1-\kappa}{\kappa}\right)}=T_{4} p_{1}^{\left(\frac{1-\kappa}{\kappa}\right)}
$$

where the appropriate temperature ratios are the same:

$$
T_{1} / T_{2}=T_{4} / T_{3} \Rightarrow T_{4}=T_{3}\left(T_{1} / T_{2}\right)
$$

The work required to drive the system is the difference between the work consumed by the compressor $\left(\mathrm{W}_{\mathrm{C}}\right)$ and the work done by the turbine $\left(\mathrm{W}_{\mathrm{T}}\right)$ :

$$
W_{M}=W_{C}-W_{T}=m c_{p}\left[\Delta T_{C}-\Delta T_{T}\right]
$$

The heat transferred through HTHX is:

$$
Q_{H}=m c_{p} \Delta T_{H}
$$


The share of mechanical work Rwm and the overall efficiency $\eta$ does not depend on the temperature span $\Delta \mathrm{T}_{\mathrm{H}}$ so the results are valid also for very narrow temperature windows. For $\mathrm{T}_{1}=530^{\circ} \mathrm{C}, \mathrm{T}_{2}=870^{\circ} \mathrm{C}$, and $\eta_{\mathrm{e}}=0.35$ the calculated performance indicators are:

- $\eta=0.64$

- $\mathrm{R}_{W M}=0.30$

- $\mathrm{R}_{\mathrm{QL}}=0.70$

These values are really much better than the ones in case of electric heater, but are valid only for the ideal cycle. In reality the processes of compression and expansion are irreversible and the pressure drop in heat exchangers and piping cannot be neglected. On the other hand, these values indicate the limits, which in fact cannot be improved.

\section{Irreversible processes}

When the processes of compression and expansion are irreversible, the temperature ratios in eq. (8) are valid for temperatures $\mathrm{T}_{2 s}$ and $\mathrm{T}_{4 \mathrm{~s}}$ in equivalent isentropic processes:

$$
T_{1} / T_{2 s}=T_{4 s} / T_{3} \Rightarrow T_{4 s}=T_{3}\left(T_{1} / T_{2 s}\right)
$$

The temperatures $\mathrm{T}_{2 \mathrm{~s}}$ and $\mathrm{T}_{4 \mathrm{~s}}$ are determined by internal efficiencies of compressor $\left(\eta_{C}\right)$ and turbine $\left(\eta_{T}\right)$ respectively:

$$
T_{2 s}-T_{1}=\eta_{C}\left(T_{2}-T_{1}\right) \quad \text { and } T_{4}-T_{3}=\eta_{T}\left(T_{4 s}-T_{3}\right)
$$

Now, the share of mechanical work RwM depends not only on the temperature span $\Delta \mathrm{T}_{\mathrm{H}}$ but also on the internal efficiencies $\eta_{C}$ and $\eta_{T}$. When the temperature span $\Delta T_{H}$ is below the limit $\Delta T_{H}^{M I N}$, the RWM $>1.0$ and the LTHX will transport heat in an opposite direction. Part of work will be lost for heating steam.

For the realistic values $\Delta T_{H}=100$ and $\eta_{C}=\eta_{T}=0.90$ the calculated performance indicators are:

- $\eta=0.40$

- $\mathrm{R}_{W M}=0.81$

- $\mathrm{R}_{\mathrm{QL}}=0.19$

The overall efficiency is still higher than it was for electric heating, but the gain is not very impressive. Simultaneously, the calculated $\Delta T_{H}^{M I N}=74.7^{\circ} \mathrm{C}$ determines the limit below which the overall efficiency will be worse than for the simple reference case.

\section{Irreversible processes and pressure drop}

The pressure drops in cycle are characterized by the parameter $\beta$ :

$$
\beta=\left(\frac{p_{4}}{p_{1}} \frac{p_{2}}{p_{3}}\right)^{\frac{\kappa-1}{\kappa}}
$$

where $\mathrm{p}_{\mathrm{i}}$ are the pressures for the respective temperatures $\mathrm{T}_{\mathrm{i}}$ and $\kappa=C_{p} / C_{v}$. With these pressure ratios it is possible to calculate the mechanical work $W_{M}$, its share $\mathrm{R}_{\mathrm{WM}}$ and the overall efficiency $\eta$. For $\beta=1.02$ ( $1 \%$ pressure drop on both LT and HT HXs), the respective performance indicators are nearly the same as for electric heating.

- $\eta=0.36$

- $\mathrm{R}_{w \mathrm{~m}}=0.95$

- $\mathrm{R}_{\mathrm{QL}}=0.05$

The conclusion is that in comparison with the simplest configuration, the system with the heat pump gets much more complex but with negligible performance improvement. 
Considering the uncertainties regarding the realistic values of internal efficiencies, pressure drops and required width of temperature window, it is clear that the simple heat pump cannot guarantee a satisfactory results.

\subsubsection{Heat pump with regeneration}

Heat pump performance can be significantly improved by using the regenerative heat exchanger (Fig. 5). Although it does not enable the recovery of waste heat, but allows adjustment of the system to a narrow temperature window. The heat at temperatures lower than required by the chemical processes is used to heat up gas leaving the LT HX. The compression starts at temperature higher than the temperature on the LT HX outlet and the compressor requires less work to get the required temperature $\mathrm{T}_{\mathrm{H}}$.

In real situations the regenerative HX (RHX) cannot pass heat at the same temperatures for both streams and some shift $\Delta \mathrm{T}_{\mathrm{o}}$ must be taken into account. The relationships between temperatures shown in Fig. 5 explain that the temperature difference $\Delta T_{R}$ at the RHX cannot be arbitrary and is determined by the assumed values of $\mathrm{T}_{\mathrm{L}}, \mathrm{T}_{\mathrm{H}}, \Delta \mathrm{T}_{\mathrm{H}}$ and $\Delta \mathrm{T}_{\mathrm{o}}$ :

$$
\Delta T_{R}=T_{H}-T_{L}-\Delta T_{H}-\Delta T_{o}
$$

Simultaneously, the work consumed by compressor depends only on the required temperature span $\Delta \mathrm{T}_{\mathrm{H}}$ and the temperature shift $\Delta \mathrm{T}_{\mathrm{o}}$ :

$$
W_{C}=m c_{p}\left(\Delta T_{H}+\Delta T_{o}\right)
$$
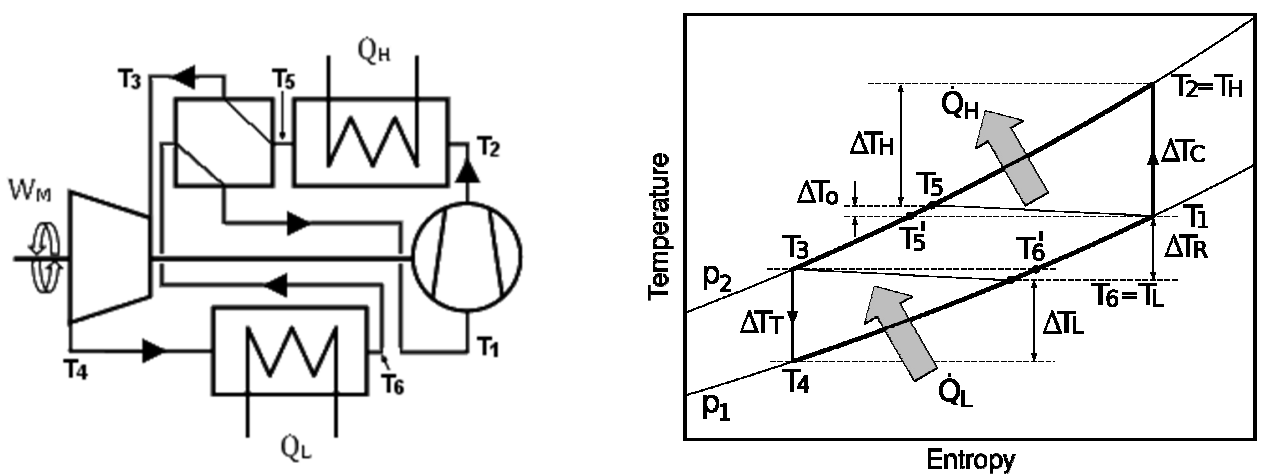

Fig. 5. HT heat pump with regenerative HX and the reverse Brayton cycle for reversible processes

The calculated performance indicators of the heat pump with a RHX are shown in Table 1 together with the results obtained in the previous case.

Table 1. Comparison of performance indicators calculated in various models.

\begin{tabular}{|l|c|c|c|c|c|c|}
\hline \multirow{2}{*}{ Model } & \multicolumn{2}{|c|}{$\eta$} & \multicolumn{2}{c|}{ RwM } & \multicolumn{2}{c|}{ RQL $^{*}$} \\
\cline { 2 - 7 } & no RHX & RHX & no RHX & RHX & no RHX & RHX \\
\hline electric heating & 0.35 & $\mathrm{x}$ & 1.00 & $\mathrm{x}$ & 0.00 & $\mathrm{x}$ \\
\hline reversible processes & 0.64 & 0.63 & 0.30 & 0.32 & 0.70 & 0.68 \\
\hline irreversible processes & 0.40 & 0.54 & 0.81 & 0.46 & 0.19 & 0.54 \\
\hline irrevers. + pressure drop & 0.36 & 0.48 & 0.95 & 0.59 & 0.05 & 0.41 \\
\hline
\end{tabular}


While the regenerative heat exchanger doesn't help much in systems with reversible processes, the greatest efficiency improvement can be observed in case of models with irreversible processes $(0.54$ vs 0.40$)$. When additionally the pressure drops are taken into account, the efficiency improvement is still good ( 0.48 vs 0.36$)$. However, it should be remembered that the pressure drops in heat exchangers depend on the fluid velocity, which for gaseous media will be high. The dependence of overall efficiencies $\eta$ on pressure drop coefficients $\beta$ calculated for three selected internal efficiencies $\eta_{\mathrm{i}}=\eta_{\mathrm{C}}=\eta_{\mathrm{T}}$ are shown in Fig. 6.

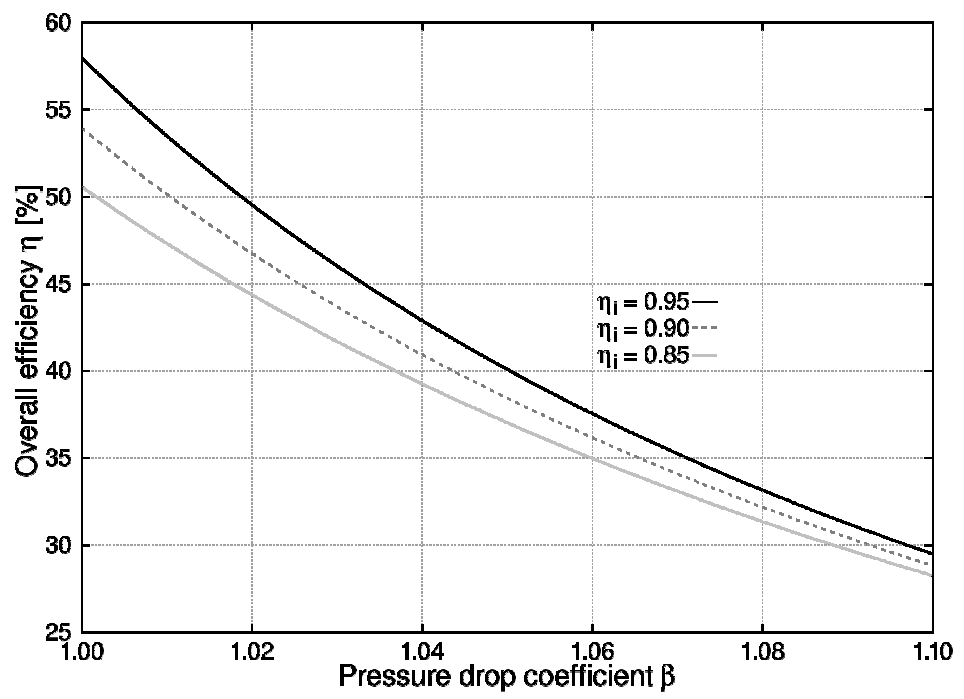

Fig. 6. Overall efficiency $\eta$ as a function of pressure drop coefficient $\beta$

\section{Key issues of HT heat pumps}

The chemical class end-users expect that the nuclear heat supply system will work reliably, supplying heat at the required temperature without any interruptions. In this context, the biggest challenges are related to the design of high-temperature heat pump capable of long term operation in temperatures above $850^{\circ} \mathrm{C}$. The key issues are related to:

- materials capable of long term operations in temperatures $870-880^{\circ} \mathrm{C}$. The existing candidate materials are Alloy 617, Haynes 230, Hasteloy X and Alloy $800 \mathrm{H}$, but only the last one is currently approved for high-temperature applications and only up to $760^{\circ} \mathrm{C}[4]$;

- compressor design. The compressor will work at highest temperatures $\left(870-880^{\circ} \mathrm{C}\right)$ while the rotating parts will cause mechanical stress. Since the highest temperature is required, the compressor cannot be cooled. These conditions are a big challenge for the compressor design;

- design of compact heat exchangers. The required gas heat exchangers must be capable of transferring large thermal power in temperatures up to $880^{\circ} \mathrm{C}$ and, at the same time, must withstand creep damage for long lifetimes;

- turbine design - will depend on the working gas. In case of helium the internal efficiency must be improved. 
Due to the above mentioned issues the design of the heat pump with the expected parameters still requires intensive $R \& D$ work, and can be considered only as a mediumterm option.

\section{Conclusions}

Coupling of HTR reactors with chemical class installations by means of steam-based heat transport loop followed by a high-temperature heat pump is possible, but shouldn't be considered as a near-term solution. Simple thermodynamic calculations show that the use of heat pumps instead of electric heating could improve overall efficiency by several percentage points, but the actual numbers depend strongly on parameters of key devices. In pessimistic scenario the overall efficiency may prove to be lower than for a simple system with electric heating. But even in the best case this efficiency will be lower than 0.66 significantly less than the result for the direct high-temperature heat transfer (1.0 when all loses omitted). At the same time it should be noted, that the biggest challenge for the construction of high-temperature heat pump will be issues with materials capable of longterm operation at temperatures of $870-880^{\circ} \mathrm{C}$. In fact, the same problems prevent the successful deployment of direct coupling by means of the previously mentioned gas loop. Progress in the field of high-temperature materials is therefore essential both to construction of the high-temperature heat pump and to construction of the direct gas-based heat transport loop. The latter solution would offer significantly higher overall efficiency, being simultaneously less complicated and easier in exploitation.

Therefore it should be concluded that in chemical-class applications the only HTR heat supply system feasible in a near term is a steam loop with electric heating. The configuration with a high-temperature heat pump is not feasible in a near term, mainly due to material issues. The construction of such an installation is likely to be possible in future, but probably will not be profitable because of availability of an alternative solution offering much better performance.

\section{References}

1. E. Bogusch, EUROPAIRS Final Report, EC FP7 (2011)

2. A. Marmier, M.A. Fütterer, Nucl. Eng. Des., 238, 2272, (2008)

3. J.M. Beck, L.F. Pincock, High Temperature Gas-Cooled Reactors Lessons Learned Applicable to the Next Generation Nuclear Plant, INL/EXT-10-19329 (2011)

4. G.A. Gibbs, NGNP Project 2011 Status and Path Forward, INL/EXT-11-23907 (2011) 